\title{
Série histórica de mortalidade por suicídio em município paulista segundo dados epidemiológicos
}

\author{
Time series of suicide mortality in a municipality in São Paulo according to \\ epidemiological data
}

\section{Serie histórica de mortalidad por suicidio del municipio de São Paulo según datos epidemiológicos}

\author{
Natalia Cristina da Silva Freire iD \\ Faculdade São Leopoldo Mandic - Campinas (SP) - Brasil
}

Taís Daiene Russo Hortencio iD

Faculdade São Leopoldo Mandic - Campinas (SP) - Brasil

Márcia Regina Campos Costa da Fonseca iD

Faculdade São Leopoldo Mandic - Campinas (SP) - Brasil

Faculdade de Medicina de Jundiaí - Jundiaí (SP) - Brasil

\section{RESUMO}

Objetivo: Analisar a tendência e o perfil epidemiológico do suicídio, em um município de grande porte do interior do estado de São Paulo, no período de 2000 a 2018. Métodos: Estudo ecológico de série temporal realizado em 2019 que analisou a mortalidade por suicídio na cidade de Campinas, São Paulo, Brasil, no período de 2000 a 2018. A coleta ocorreu por meio de dados do Sistema de Informação sobre Mortalidade, sendo as variáveis: sexo, idade, raça, categoria do óbito, mês e local de ocorrência, com dados analisados pelo número/taxa de óbitos e taxas de óbitos padronizadas por faixa etária e sexo. Estimaramse modelos de regressão linear simples para número de óbitos e ano. Na análise da proporção de óbitos por suicídio e demais variáveis estudadas utilizou-se teste de qui-quadrado com significância de 5\%. Resultados: Ocorreram 904 óbitos por suicídio, com aumento significativo ao longo dos anos e taxa geral de 3,20/100.000 hab. (2000) e 5,42/100.000 hab. (2018). Observou-se aumento no sexo masculino 5,30/100.000 hab. (2000) e 8,45/100.000 hab. (2018), faixas etárias de 20 a 40 incompletos com 3,53/100.000 hab. (2000) e 6,84/100.000 hab. (2018) e de 40 a 60 incompletos com 4,69/100.000 hab. (2000) e 7,61/100.000 hab. (2018). A maioria dos óbitos ocorreu com pessoas brancas (673; 74,9\%), por enforcamento (503; 55,6\%), em domicílio (524; $58,0 \%)$, em setembro $(93 ; 10,3 \%)$ e dezembro $(92 ; 10,2 \%)$. Conclusão: Houve crescente aumento das taxas de suicídios em Campinas no período analisado, sendo as vítimas predominantemente homens, adultos, brancos, por enforcamento e realizado em domicílios.

Descritores: Mortalidade; Suicídio; Estudos de Séries Temporais; Epidemiologia; Saúde Pública.

\begin{abstract}
Objective: To analyze the trend and epidemiological profile of suicide in a large municipality in the countryside of the state of São Paulo from 2000 to 2018. Methods: An ecological time-series study was conducted in 2019 to analyze suicide mortality in the city of Campinas, São Paulo, Brazil, in the period from 2000 a 2018. Data were extracted from the Mortality Information System, with the following variables being investigated: sex, age, race, death category, month, and place of occurrence. The data were analyzed by number of deaths/death rates and by the standardized death rates for age range and sex. Simple linear regression models were estimated for the number of deaths and year. In the analysis of the rate of deaths by suicide for the other study variables, the chi-square test was used with a significance level of 5\%. Results: In the analyzed period, there were 904 deaths from suicide, with a significant increase over the years and an overall rate of 3.20/100,000 inhabitants (2000) and 5.42/100,000 inhabitants (2018). A significant increase was also observed for males, with 5.30/100,000 inhabitants (2000) and 8.45/100,000 inhabitants (2018), age ranges from 20 to incomplete 40 years, with 3.53/100,000 inhabitants (2000) and 6.84/100,000 inhabitants (2018), and 40 to incomplete 60 years, with 4.69/100,000 inhabitants (2000) and 7.61/100,000 inhabitants (2018). Most deaths occurred among White people (673; 74.9\%), by hanging (503; 55.6\%), at home (524; 58.0\%), in September (93; 10.3\%) and December (92; 10.2\%). Conclusion: There was a growing increase in suicide rates in Campinas in the period analyzed, with the victims being predominantly males, adults, White individuals, and deaths by hanging and occurring at home.
\end{abstract}

Descriptors: Mortality; Suicide; Time Series Studies; Epidemiology; Public Health. 


\section{RESUMEN}

Objetivo: Analizar la tendencia y el perfil epidemiológico del suicidio de un gran municipio del interior del estado de São Paulo en el período entre 2000 y 2018. Métodos: Estudio ecológico de serie temporal realizado en 2019 que analizó la mortalidad por suicidio de la ciudad de Campinas, São Paulo, Brasil, en el periodo entre 2000 y 2018. La recogida de datos se dio a través del Sistema de Información de Mortalidad con las siguientes variables investigadas: sexo, edad, raza, categoría del óbito, mes y sitio de ocurrencia. Se analizaron los datos por el número/ la tasa de óbitos y las tasas de óbitos estandarizadas por la franja de edad y el sexo. Se ha estimado modelos de regresión linear simple para el numero de óbitos y el año. Se utilizó la prueba de chi-cuadrado para el análisis de la proporción de óbitos por suicidio y las demás variables con el nivel de significación del 5\%. Resultados: En el período analizado ocurrieron 904 óbitos por suicidio con el aumento significativo a lo largo de los años y la tasa general de 3,20/100.000 hab. (2000) y 5,42/100.000 hab. (2018). Se observó también el aumento significativo para el sexo masculino de 5,30/100.000 hab. (2000) y 8,45/100.000 hab. (2018), las franjas de edad entre los 20 y los 40 años incompletos de 3,53/100.000 hab. (2000) y 6,84/100.000 hab. (2018) y entre los 40 y los 60 incompletos de 4,69/100.000 hab. (2000) y 7,61/100.000 hab. (2018). La mayoría de los óbitos se dio de personas del color blanco (673; 74,9\%), por ahorcamiento (503; $55,6 \%)$, en el domicilio (524; 58,0\%), en septiembre (93; 10,3\%) y diciembre (92; 10,2\%). Conclusión: Hubo un creciente aumento de las tasas de suicidios de Campinas en el período analizado de víctimas predominantemente hombres, adultos, blancos, por ahorcamiento y realizado en sus domicilios.

Descriptores: Mortalidad; Suicidio; Estudios de Series Temporales; Epidemiología; Salud Pública.

\section{INTRODUÇÃO}

O suicídio é definido como a ação do indivíduo de tirar sua própria vida de maneira intencional e voluntária, ou seja, ato de matar-se deliberadamente ${ }^{(1-3)}$. Sendo, infelizmente, o suicídio um fenômeno mundial, dados da Organização Mundial de Saúde (OMS) revelam que, anualmente, cerca de 800.000 mil pessoas morrem devido ao suicídio todos os anos, resultando em uma taxa média global de 10,6 por 100.000 habitantes (15,0 para homens e 8,0 para mulheres) $)^{(1)}$, com previsão de aumento nas próximas décadas ${ }^{(3)}$.

Segundo a OMS, $78 \%$ dos suicídios globais ocorrem em países de baixa e média renda(1), sendo o Brasil o oitavo país em número absoluto de suicídios $^{(4)}$. O último boletim divulgado pelo Ministério da Saúde, de 2011 a 2017, registrou 80.352 óbitos por suicídio na população a partir de 10 anos, dos quais $21.790(27,3 \%)$ ocorreram na faixa etária de 15 a 29 anos, com o número de $17.221(79,0 \%)$ no sexo masculino ${ }^{(5)}$. Dessa forma, comparando as taxas apresentadas no boletim epidemiológico de $2017^{(6)}$ e o último dado disponível no Sistema de Informação sobre Mortalidade (SIM) as taxas para 2011, 2015 e 2019 apresentaram-se, respectivamente, 5,3/100 mil hab., $5,7 / 100$ mil hab. e 6,4/100 mil hab.(7).

O Brasil e outros países, orientados pelo Plano de Ação de Saúde Mental da $\mathrm{OMS}^{(8)}$ se comprometeram a trabalhar com uma redução de 10\% em sua taxa de suicídio até 2020 (considerando as taxas de suicídio em 2012 ou 2013 como baseline). Estudo realizado com o objetivo de analisar a tendência histórica das taxas de suicídio no Brasil e regiões (1997-2015) e comparar as taxas estimadas de suicídio projetadas para 2020, com a meta do Plano de Ação em Saúde Mental da Organização Mundial da Saúde (OMS), concluiu que a maior parte dos estados brasileitos apresentou tendência estável ou crescente nas taxas e que não alcançariam a redução recomendada pela OMS até $2020^{(9)}$.

A distribuição epidemiológica do suicídio depende de um conjunto complexo de fatores, incluindo os sociodemográficos, genéticos, psicológicos, culturais, sociais e econômicos ${ }^{(1,2,8,10)}$. Observa-se que pessoas que cometeram suicídio, na maior parte das vezes, tinham transtornos mentais diagnosticáveis, e que o comportamento suicida e o suicídio apresentam-se mais frequentes em indivíduos com depressão, com transtorno de personalidade, alcoólatras e esquizofrênicos ${ }^{(2,11)}$. Há também doenças físicas que estão associadas ao suicídio, como as neurológicas (epilepsia, trauma medular ou craniano e acidente vascular cerebral), câncer, HIV e AIDS ${ }^{(11)}$.

Os fatores de risco para o suicídio vão além das questões individuais, como os associados com o sistema de saúde e sociedade, onde se destaca as dificuldades de acesso para obtenção à atenção em saúde e receber assistência necessária; a fácil disponibilidade de meios utilizados para suicidar-se; o sensacionalismo dos meios de comunicação em relação à temática, aumentando o risco de imitação de atos suicidas; além da dificuldade, gerada pelo estigma, para aqueles que querem buscar ajuda(1). No entanto, o suicídio pode, pelo menos parcialmente, ser evitado restringindo o acesso aos meios de suicídio, treinando médicos e profissionais da atenção primária para identificar pessoas em risco, avaliar e gerenciar as crises e fornecer acompanhamento adequado(11,12), e abordar a forma como o fenômeno é relatado pela mídia ${ }^{(12)}$. 
Para que as respostas nacionais sejam eficazes, é necessária uma estratégia abrangente de prevenção multissetorial do suicídio focada em promoção de saúde mental. Esta ação requer colaboração de vários setores da sociedade, públicos e privados, incluindo saúde, educação, trabalho, justiça, entre outros, assim como os responsáveis pelas leis, mídia e política ${ }^{(1)}$. O Brasil, preocupado com a expansão do suicídio, em 2006, lançou a $1^{\text {a }}$ Portaria que trata do assunto, instituindo as Diretrizes Nacionais para Prevenção do Suicídio, norteando as estratégias do Ministério da Saúde para atuação e contenção do fenômeno ${ }^{(13)}$. Posteriormente à esta Portaria, desenvolveram-se outras iniciativas visando sistematizar as condutas a serem tomadas pelos serviços de saúde, o aprimoramento do acesso e acolhimento e acompanhamento dos indivíduos em todos os níveis de atenção à saúde ${ }^{(4)}$. Em 2006, destaca-se a criação do manual dirigido a profissionais das equipes de saúde mental, com orientações de como realizar detecção precoce de condições associadas ao comportamento suicida, manejo inicial de pessoas que se encontrem sob risco e medidas de prevenção. Em 2011 criou-se a Portaria $n^{\circ}$ 3.088/2011, que instituiu a Rede de Atenção Psicossocial (RAPS); que tem por finalidade a criação, ampliação e articulação de pontos de atenção à saúde mental para grupos populacionais de maior vulnerabilidade ao agravo, com serviços que incluem desde acompanhamento e orientação na atenção básica, até atendimentos de urgência e emergência, sob a coordenação dos Centros de Atenção Psicossocial (CAPS). Em 2017, fez-se a criação de agenda de ações estratégicas para a Vigilância e Prevenção do Suicídio e Promoção da Saúde no Brasil entre 2017 e 2020, com objetivo de ampliar e fortalecer as ações de promoção da saúde, vigilância, prevenção e atenção integral relacionadas ao suicídio. Ainda em 2017, a Portaria n 3.479 instituiu o comitê para a elaboração e operacionalização do Plano Nacional de Prevenção do Suicídio no Brasil, em consonância com as Diretrizes Nacionais para Prevenção do Suicídio e junto com as Diretrizes Organizacionais das Redes de Atenção à Saúde, que tinha entre seus objetivos, realizar diagnósticos situacionais acerca da situação do país quanto ao suicídio e seus resultantes, articular a implementação do Plano Nacional de Prevenção do Suicídio no Brasil, bem como de realizar monitoramento e avaliação do mesmo(4).

A realização de diagnósticos situacionais sobre o suicídio e o estabelecimento de hipóteses sobre a problemática, tais como: população de maior vulnerabilidade, lugar de maior ocorrência e comportamento em relação ao tempo é fundamental para subsidiar o planejamento de ações preventivas nos diversos pontos da rede de atenção à saúde, em especial na atenção primária.

Assim, este estudo teve como objetivo analisar a tendência e o perfil epidemiológico do suicídio, em um município de grande porte do interior do estado de São Paulo, no período de 2000 a 2018.

\section{MÉTODOS}

Trata-se de um estudo ecológico de série temporal, realizado em 2019 , que analisou a mortalidade por suicídio em Campinas, São Paulo, Brasil, nos anos de 2000 a 2018. Selecionaram-se dados sobre o agravo ocorridos no referido município, sendo estes provenientes do Sistema de Informação sobre Mortalidade (SIM) disponibilizado pelo tabulador de dados (TABNET-DATASUS) da Secretaria Municipal de Saúde de Campinas ${ }^{(14)}$. Selecionou-se o município pelo fato de os pesquisadores estarem vinculados à instituição de ensino de graduação de medicina na cidade de Campinas, e estes, terem atuação na rede de atenção à saúde municipal. O tabulador de dados do município gera desagregação até os centros de saúde de residência, então, além do conhecimento do perfil epidemiológico deste agravo é possível identificar áreas de maior vulnerabilidade e que merecem maior atenção dos pesquisadores, para o desenvolvimento de ações de promoção de saúde. Segundo dados do Instituto Brasileiro de Geografia e Estatística (IBGE), Campinas está localizada a 99 quilômetros a noroeste da capital do estado de São Paulo, possui população estimada (2020) de 1.213 .792 hab., área territorial de $794.571 \mathrm{~km}^{2}$ e densidade demográfica de $1.359,60$ hab./km²(15).

A determinação da série histórica (2000 - 2018) se deu em função dos anos que estavam disponíveis no SIM na época da coleta de dados (ano completo), em julho de 2019. Optou-se, assim, pela coleta de dados neste mês por julgar ser tempo hábil para possíveis correções nos dados já digitados. No TABNET - DATASUS Campinas, a base de dados é atualizada mensalmente; e podem ser feitas correções nos dados já digitados, assim como inclusão de novos registros; por esse motivo, as informações obtidas no banco de dados estão sujeitas à revisão, podendo ser modificadas e atualizadas.

Para a realização do estudo, selecionaram-se os óbitos cuja causa básica tenham sido "lesões autoprovocadas intencionalmente" (X60-X84), segundo a Classificação Estatística Internacional de Doenças e Problemas Relacionados à Saúde $-10^{a}$ Revisão (CID 10)(16), sendo investigadas as variáveis: sexo, idade, raça/cor da pele, categoria do óbito (CID 10), mês e local de ocorrência. 
A variável idade apresentou-se estratificada por faixas etárias, a saber: adolescência $(<20$ anos), fase adulta jovem (de 20 a 40 anos incompletos), meia idade (de 40 a 60 anos incompletos) e idosa/velhice ( $\geq 60$ anos), classificações esta, que leva em conta as etapas do ciclo vital e características de cada fase ${ }^{(17)}$.

Coletaram-se as variáveis selecionadas para este estudo por uma das pesquisadoras, com experiência em pesquisas com fontes secundárias e, em especial, com dados gerados pelas informações do Departamento de Informática do Sistema Único de Saúde (DATASUS). Além disso, houve uma segunda verificação dos dados para checagem da veracidade destes, realizada pela própria pesquisadora, que, posteriormente, armazenaram-se em planilha do programa de software Excel, até envio para análise estatística.

Inicialmente, analisaram-se os dados dos números de óbitos (absolutos) extraídos do SIM e as taxas brutas de mortalidade por 100.000 hab., estas, calculadas levando-se em consideração o número de óbitos da população residente em determinado momento dividido pela população total residente no mesmo período temporal ${ }^{(18)}$.

A quantificação da população residente (denominador das taxas) se deu por meio dos dados extraídos do TABNET-DATASUS Campinas, item "Demografia", sendo estas estimativas censitárias fornecidas pela Fundação Sistema Estadual de Análises de Dados (SEADE)(14), o qual fornece dados da população por sexo e faixa etária.

A seguir, padronizaram-se as taxas de óbitos por faixa etária e sexo pelo método direto ou de base fixa(19), considerando a população do Brasil do ano de 2010 (Censo) como referência ${ }^{(20)}$. O ajuste da taxa de mortalidade permite minimizar o efeito de diferenças etárias e de sexo entre populações (ou na mesma população em períodos distintos), a fim de que diferenças temporais não possam ser atribuídas a estas características.

Posteriormente, realizou-se a análise de regressão linear simples para o número de óbitos e taxas em função do ano(21). Estimaram-se modelos de regressão $Y=\alpha+\beta X$, onde $Y$ é a variável de resposta (óbitos), $X$ é o ano, $\alpha$ é o coeficiente linear, o intercepto, ou seja, o ponto onde a reta corta o eixo das ordenadas (eixo vertical) e $\beta$ é a inclinação da reta. Testaram-se as hipóteses nulas $\beta=0$, sabendo-se que um $\beta$ estatisticamente significativo e positivo indica tendência de crescimento do óbito no tempo e um $\beta$ estatisticamente significativo e negativo indica tendência de decréscimo do óbito no tempo. Calcularam-se também os coeficientes de determinação dos modelos, ou seja, o quanto da variação na quantidade de óbitos é explicada pelo modelo de regressão estimado. Esta medida pode variar de zero a um e é utilizada como uma indicação da adequação do modelo de regressão, dessa forma, portanto, estimaram-se modelos para população geral e por faixa etária e sexo.

Para analisar se a proporção de óbitos por suicídio difere entre os meses do ano, raça/cor, categoria do óbito (CID10) e local de ocorrência utilizou-se o teste de qui-quadrado para aderência ${ }^{(21)}$. As hipóteses nulas testadas apresentaram-se como H0: $p 1=p 2, \ldots ., p k$, sendo $p$ a probabilidade de cada categoria $i, i=1, \ldots, k$. Por essa análise compararam-se as proporções de óbitos nos diferentes meses do ano, diferentes raças/cor, CID10 e local de ocorrência no decorrer da série histórica, sendo as hipóteses nulas testadas para cada variável: H0 a proporção de óbitos por meses do ano não varia conforme o ano; $\mathrm{HO}$ a proporção de óbitos por raça/cor não varia conforme o ano; H0 a proporção de óbitos por CID10 não varia conforme o ano; H0 a proporção de óbitos por local de ocorrência não varia conforme $o$ ano. A partir desses dados, compreende-se, então, que as análises foram realizadas no programa $\mathrm{R}^{\star(22)}$, com nível de significância de $5 \%$.

Sabe-se que os dados do SIM se encontram divulgados e disponibilizados no TABNET-DATASUS, portanto, são de domínio público e sem a permissão ao acesso da identidade das vítimas, cujos registros constam nos bancos analisados. Além disso, e para concluir, afirma-se a dispensa deste projeto por parte do Comitê de Ética em Pesquisa pela Faculdade São Leopoldo Mandic (Parecer $n^{\circ}$. 2018/009).

\section{RESULTADOS}

Entre o período de 2000 a 2018 registraram-se em Campinas 904 óbitos por suicídio com aumento expressivo ao longo dos anos tanto em números absolutos, quanto em taxa de óbitos. O aumento pode ser observado na Tabela I pelo $p$-valor $(p<0,000)$ e pelos coeficientes angulares que são positivos, ou seja, rejeitam-se as hipóteses nulas de que esses coeficientes são iguais a zero, além do fato de serem positivos quanto ao indicativo de aumento significativo de óbitos por suicídio no decorrer do tempo.

Na comparação do início (2000), meio (2009) e fim (2018) da série histórica analisada, em relação aos números de óbitos (absolutos) e taxas de óbitos (taxas brutas de mortalidade), observou-se respectivamente: 31, 40 e 65 ( $p<0,000$ ); 3,20/100.000 hab., 3,73/100.000 hab. e 5,42 em 100.000 hab. ( $p<0,0001$ ). Nota-se também aumento relevante nas taxas de mortalidade padronizadas no decorrer do tempo, sendo a taxa geral de suicídio padronizada por sexo de 3,18/100.000 hab. em 2000, 3,69/100.000 hab. em 2009 e de 5,41/100.000 hab. em 2018 ( $p<0,0001$ ); 
padronizada por faixa etária de 3,54/100.000 hab. em 2000, 4,26/100.000 hab. em 2009 e de 5,46/100.000 hab. em 2018 ( $p=0,0008$ ) e padronizada por sexo e faixa etária de 3,53/100.000 hab. em 2000, 4,21/100.000 hab. em 2009 e de 5,45/100.000 hab. em 2018 ( $p=0,0007$ ). (Tabela I).

Tabela I - Número de óbitos e taxas de óbitos por suicídio (óbitos por 100.000 habitantes) na cidade de Campinas em função do ano (2000 a 2018). Campinas, São Paulo, 2019.

\begin{tabular}{cccccc}
\hline & & \multicolumn{4}{c}{ Taxas (óbitos por 100.000 habitantes) } \\
\cline { 3 - 6 } Ano & óbitos & $\begin{array}{c}\text { Não } \\
\text { padronizada }\end{array}$ & $\begin{array}{c}\text { Padronizada por } \\
\text { sexo }\end{array}$ & $\begin{array}{c}\text { Padronizada por } \\
\text { faixa etária }\end{array}$ & $\begin{array}{c}\text { Padronizada por } \\
\text { sexo e faixa etária }\end{array}$ \\
\cline { 3 - 6 } $\mathbf{2 0 0 0}$ & 31 & 3,20 & 3,18 & 3,54 & 3,53 \\
$\mathbf{2 0 0 1}$ & 34 & 3,47 & 3,45 & 3,71 & 3,69 \\
$\mathbf{2 0 0 2}$ & 36 & 3,63 & 3,61 & 3,81 & 3,79 \\
$\mathbf{2 0 0 3}$ & 36 & 3,59 & 3,56 & 3,84 & 3,82 \\
$\mathbf{2 0 0 4}$ & 31 & 3,05 & 3,02 & 3,40 & 3,37 \\
$\mathbf{2 0 0 5}$ & 35 & 3,40 & 3,39 & 3,83 & 3,82 \\
$\mathbf{2 0 0 6}$ & 42 & 4,04 & 4,02 & 4,51 & 4,49 \\
$\mathbf{2 0 0 7}$ & 52 & 4,95 & 4,92 & 5,56 & 5,52 \\
$\mathbf{2 0 0 8}$ & 57 & 5,37 & 5,35 & 5,96 & 5,94 \\
$\mathbf{2 0 0 9}$ & 40 & 3,73 & 3,69 & 4,26 & 4,21 \\
$\mathbf{2 0 1 0}$ & 53 & 4,91 & 4,91 & 4,91 & 4,91 \\
$\mathbf{2 0 1 1}$ & 52 & 4,77 & 4,77 & 4,77 & 4,77 \\
$\mathbf{2 0 1 2}$ & 46 & 4,17 & 4,17 & 4,18 & 4,18 \\
$\mathbf{2 0 1 3}$ & 49 & 4,40 & 4,40 & 4,41 & 4,41 \\
$\mathbf{2 0 1 4}$ & 58 & 5,16 & 5,16 & 5,17 & 5,17 \\
$\mathbf{2 0 1 5}$ & 64 & 5,64 & 5,63 & 5,66 & 5,66 \\
$\mathbf{2 0 1 6}$ & 58 & 4,94 & 4,94 & 4,96 & 4,96 \\
$\mathbf{2 0 1 7}$ & 65 & 5,47 & 5,47 & 5,50 & 5,50 \\
$\mathbf{2 0 1 8}$ & 65 & 5,42 & 5,41 & 5,46 & 5,45 \\
Regressão linear: & & & & & \\
Coeficiente linear $(\boldsymbol{\alpha})$ & $-3741,32$ & $-247,87$ & $-250,84$ & $-200,24$ & $-202,97$ \\
Coeficiente angular $(\boldsymbol{\beta})$ & 1,89 & 0,12 & 0,13 & 0,10 & 0,10 \\
$\mathbf{R}^{\mathbf{2}}$ & 0,81 & 0,66 & 0,68 & 0,51 & 0,52 \\
p-valor & $<0,000$ & $<0,0001$ & $<0,0001$ & 0,0008 & 0,0007 \\
\hline
\end{tabular}

Fonte: SIM: Sistema de Informação sobre Mortalidade; TABNET-SMS. Campinas, São Paulo. *óbitos por 100.000 habitantes

Estimaram-se, então, modelos de regressão linear $Y$ (óbitos) $=\alpha+\beta \times$ ano. $R^{2}$ : Coeficiente de determinação do modelo de regressão (pode variar de 0 a 1, quanto mais próximo de 1, melhor é o ajuste do modelo) e hipóteses nulas testadas $\mathrm{H}: \beta=0$.

A análise das taxas de óbitos padronizados para cada sexo e faixa etária é apresentada na Tabela II. Diante do grande volume de dados, optou-se pela apresentação em tabela apenas das taxas padronizadas, porém, analisaram-se também as taxas brutas de mortalidade por sexo e faixa etária. Nessas análises, portanto, para o sexo masculino observou-se aumento significativo na taxa no decorrer do tempo, de 5,30/100.000 hab. em 2000, 6,66/100.000 hab. em 2009 e 8,45/100.000 hab. em 2018 ( $p<0,0001$ ). Observa-se, então, que esse aumento de $1,21 / 100.000$ hab. em 2000, 0,91/100.000 hab. em 2009 e 2,58/100.000 hab. em 2018 ( $p=0,4758)$ não se apresenta como relevante para o sexo feminino.

Em relação à faixa etária, embora as maiores taxas tenham sido observadas em idosos (7,55/100.000 hab. em $2000,8,36 / 100.000$ hab. em 2009 e 8,26/100.000 hab. em 2018), essas variações não se apresentaram expressivas no decorrer do tempo $(p=0,1188)$. Já entre as faixas etárias de 20 a 40 anos incompletos $(3,53 / 100.000$ hab. em 2000, 4,76/100.000 hab. em 2009 e 6,84/100.000 hab. em 2018, p=0,0091) e nas faixas etárias de 40 a 60 anos incompletos (4,69/100.000 hab. em 2000, 6,08/100.000 hab. em 2009 e 7,61/100.000 hab. em 2018, p=0,0335) houve crescimento significativo no período da série histórica. Nos adolescentes ( $<20$ anos) também não se observou variação considerável no tempo, com taxas de mortalidade bruta de 0,62/100.000 hab. em 2000, nenhum registro em 2009 e 0,31/100.000 em 2018 ( $p=0,7746)$. (Tabela II). 
$\mathrm{Na}$ análise das taxas de óbitos padronizados, considerando-se o sexo e a faixa etária, verificou-se que o aumento significativo, ao longo dos anos, ocorreu entre os homens na faixa de 20 a 40 anos incompletos (5,97/100.000 hab. em 2000, 8,55/100.000 hab. em 2009 e 11,03/100.000 hab. em 2018, p=0,0027) e de 40 a 60 anos incompletos (6,89/100.000 hab. em 2000, 11,75/100.000 hab. em 2009, e 10,51/100.000 hab.em 2018, p=0,0110) (Tabela II). Esses aumentos significativos são observados pelos p-valores que estão abaixo do nível de significância estabelecido $(p<0,05)$ e pelos coeficientes angulares positivos.

Tabela II - Taxa padronizada de óbitos por suicídio (óbitos por 100.000 habitantes) na cidade de Campinas, São Paulo, em função do ano (2000 a 2018) para sexo e faixa etária. Campinas, São Paulo, 2019.

\begin{tabular}{|c|c|c|c|c|c|c|c|c|}
\hline \multirow{2}{*}{ Ano } & \multicolumn{4}{|c|}{ Sexo masculino } & \multicolumn{4}{|c|}{ Sexo feminino } \\
\hline & $<20$ anos & 20 a 40 anos & 40 a 60 anos & $\geq 60$ anos & $<20$ anos & 20 a 40 anos & 40 a 60 anos & $\geq 60$ anos \\
\hline 2000 & 1,23 & 5,97 & 6,89 & 15,06 & 0,00 & 1,16 & 2,69 & 1,89 \\
\hline 2001 & 1,21 & 7,66 & 7,80 & 7,50 & 0,62 & 2,29 & 1,78 & 1,88 \\
\hline 2002 & 2,38 & 6,39 & 7,73 & 9,95 & 1,21 & 2,83 & 1,76 & 0,00 \\
\hline 2003 & 2,34 & 6,30 & 6,67 & 9,85 & 0,60 & 2,80 & 1,76 & 3,76 \\
\hline 2004 & 0,58 & 4,53 & 12,31 & 9,87 & 0,00 & 2,21 & 0,87 & 0,00 \\
\hline 2005 & 0,57 & 5,59 & 9,38 & 4,91 & 0,00 & 1,64 & 5,16 & 5,59 \\
\hline 2006 & 0,56 & 7,18 & 9,30 & 7,34 & 0,00 & 3,24 & 5,13 & 5,58 \\
\hline 2007 & 0,55 & 8,74 & 11,98 & 19,49 & 0,56 & 3,21 & 5,09 & 1,85 \\
\hline 2008 & 0,55 & 10,26 & 7,27 & 16,86 & 0,56 & 4,24 & 10,10 & 1,85 \\
\hline 2009 & 0,00 & 8,55 & 11,75 & 14,48 & 0,00 & 1,05 & 0,83 & 3,69 \\
\hline 2010 & 1,36 & 12,27 & 7,74 & 12,33 & 0,00 & 3,09 & 3,44 & 0,00 \\
\hline 2011 & 0,67 & 11,08 & 5,37 & 17,50 & 0,69 & 3,57 & 2,04 & 2,58 \\
\hline 2012 & 1,99 & 3,13 & 11,39 & 15,64 & 0,68 & 2,52 & 4,05 & 1,28 \\
\hline 2013 & 1,31 & 10,86 & 10,53 & 6,90 & 0,68 & 1,50 & 1,34 & 2,54 \\
\hline 2014 & 0,65 & 10,75 & 10,43 & 18,84 & 0,67 & 2,97 & 1,99 & 1,26 \\
\hline 2015 & 1,28 & 10,64 & 14,02 & 17,01 & 0,00 & 2,94 & 1,31 & 5,02 \\
\hline 2016 & 1,23 & 10,29 & 12,16 & 11,61 & 1,27 & 1,89 & 2,55 & 1,23 \\
\hline 2017 & 1,22 & 11,64 & 14,16 & 11,37 & 0,63 & 0,94 & 4,42 & 2,41 \\
\hline 2018 & 0,60 & 11,03 & 10,51 & 16,16 & 0,00 & 2,78 & 5,01 & 2,40 \\
\hline \multicolumn{9}{|l|}{$\begin{array}{l}\text { Regressão } \\
\text { linear: }\end{array}$} \\
\hline $\begin{array}{l}\text { Coeficiente } \\
\text { linear }(\alpha)\end{array}$ & 41,07 & $-616,83$ & $-509,27$ & $-567,51$ & $-16,17$ & 7,08 & $-64,27$ & $-18,12$ \\
\hline $\begin{array}{l}\text { Coeficiente } \\
\text { angular }(\beta)\end{array}$ & $-0,02$ & 0,31 & 0,26 & 0,29 & 0,01 & $-0,01$ & 0,03 & 0,01 \\
\hline $\mathbf{R}^{2}$ & 0,0303 & 0,42 & 0,32 & 0,14 & 0,01 & 0,01 & 0,01 & 0,01 \\
\hline$p$-valor & 0,5180 & 0,0027 & 0,0110 & 0,1166 & 0,6561 & 0,9526 & 0,7328 & 0,8869 \\
\hline
\end{tabular}

Fonte: SIM: Sistema de Informação sobre Mortalidade; TABNET-SMS. Campinas, São Paulo. Estimou-se modelos de regressão linear $Y$ (taxa de óbitos) $=\alpha+\beta x$ ano. $R^{2}$ : Coeficiente de determinação do modelo de regressão (pode variar de 0 a 1 , pois quanto mais próximo de 1 , melhor é o ajuste do modelo). Hipóteses nulas testadas $\mathrm{H}: \beta=0$

Em relação à variável sociodemográfica "raça/cor", os resultados são apresentados como proporção (teste quiquadrado), uma vez que no Censo Demográfico só há estratificação da população por sexo e idade, portanto, não há disponibilidade do denominador para o cálculo das taxas.

Observou-se que a maior porcentagem de óbitos por suicídio ocorreu na raça/cor branca representando $77,4 \%$ de todos os casos em 2000, 82,5\% em 2009 e76,6\% em 2018. Em relação à parda, 19,4\% de todos os casos em 2000, 12,5\% em 2009 e 18,8\% em 2018. Para a negra, 3,2\% em 2000, 5,0\% em 2009 e 3,1\% em 2018 Já para amarela não ocorreram casos em 2000 e 2009, sendo1,6\% de todos os casos em 2018. Do total (entre os anos de 2000-2018) 74,9\% dos óbitos ocorreram na raça/cor branca, sendo a proporção de óbitos para as raças pardas, negras e amarelas respectivamente de: $19,0 \%, 4,8 \%$ e 1,2\% (Figura 1). 


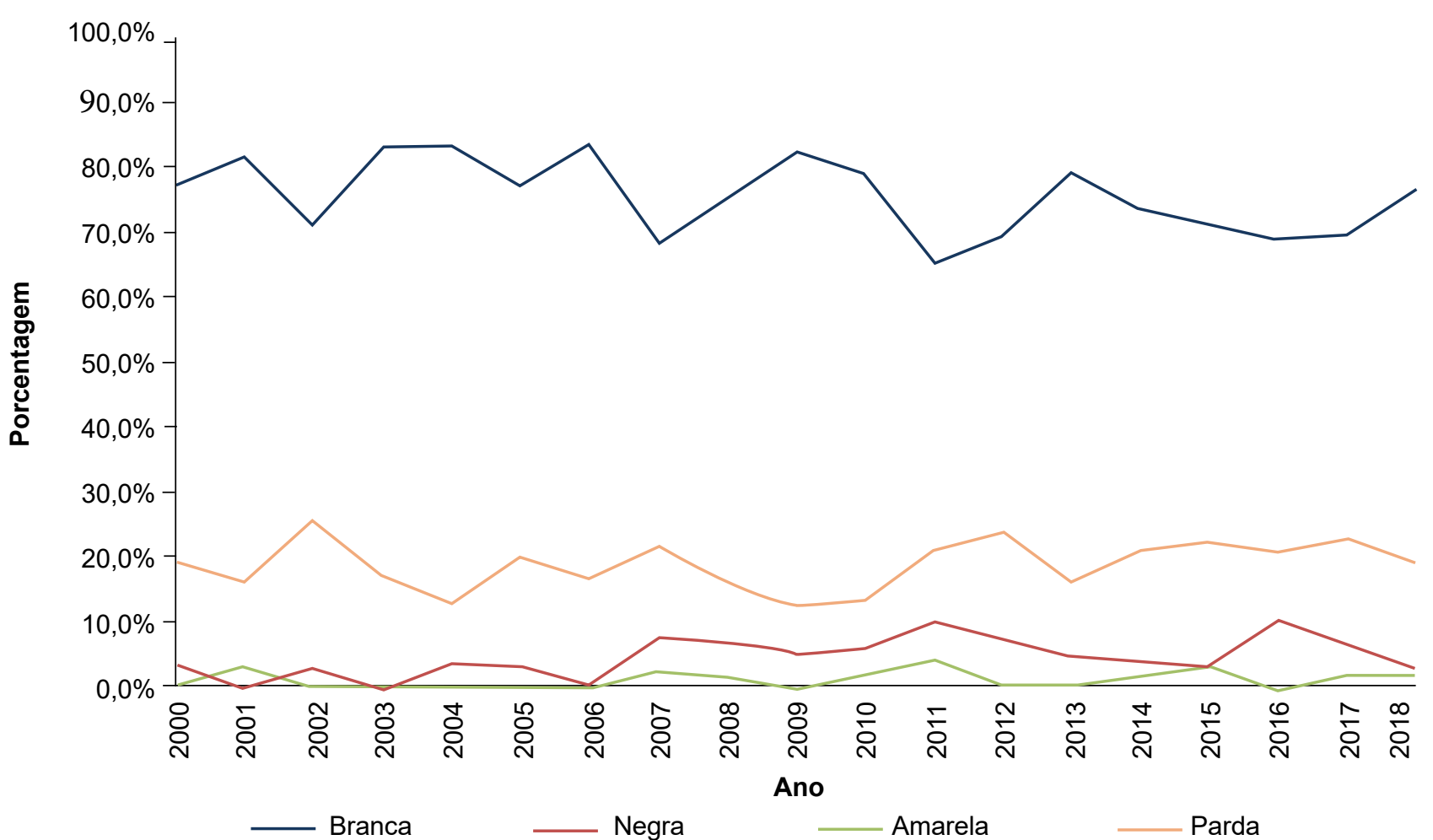

Fonte: SIM: Sistema de Informação sobre Mortalidade, TABNET- SMS. Campinas, São Paulo.

Figura 1 - Porcentagem de óbitos por suicídio na cidade de Campinas, São Paulo, em função da raça/cor e ano (2000 a 2018). Campinas, São Paulo, 2019.

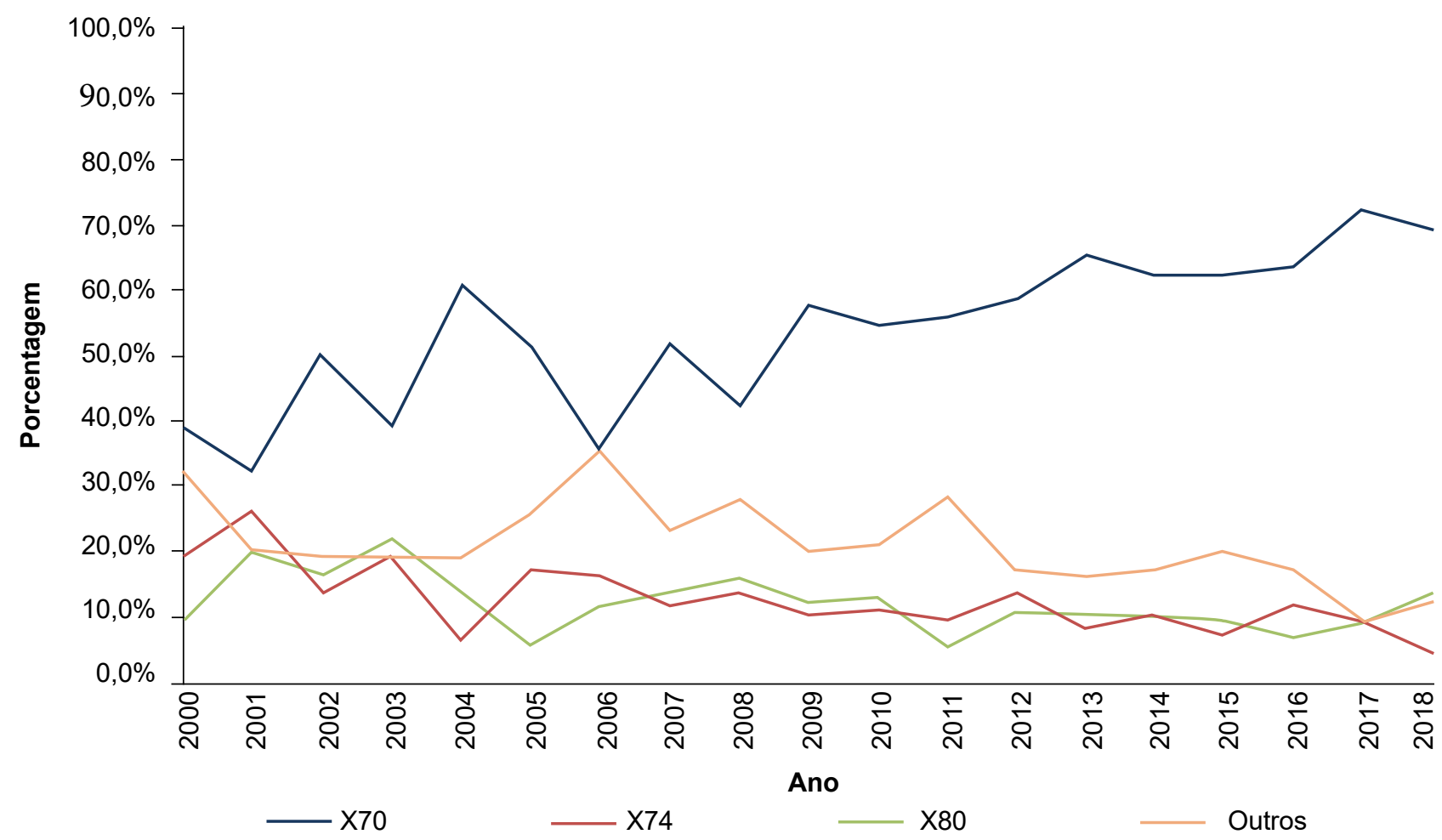

Fonte: SIM: Sistema de Informação sobre mortalidade, TABNET- SMS. Campinas, São Paulo.

*: X70: Lesão autoprovocada intencionalmente por enforcamento, estrangulamento e sufocação; X74: Lesão autoprovocada intencionalmente por disparo de outra arma de fogo e de arma de fogo não especificada; X80: Lesão autoprovocada intencionalmente por precipitação de um lugar elevado

Figura 2 - Porcentagem de óbitos por suicídio na cidade de Campinas-São Paulo, em função das categorias CID 10 mais frequentes*e do ano (2000 a 2018). Campinas, São Paulo, 2019. 
Quanto à categoria dos óbitos (CID 10), a Figura 2 chama a atenção para as mais frequentes. "Lesão autoprovocada intencionalmente por enforcamento, estrangulamento e sufocação" (X70) ocupou o primeiro lugar, sendo, 38,7\% em 2000, 57,5\% em 2009 e 69,2\% em 2018. No item "outros" agrupou-se as diversas categorias do CID $10 \mathrm{com}$ números muito pequenos, sendo 32,3\% em 2000, 20,0\% em 2009 e 12,3\% em 2018. Na sequência, vieram os óbitos por "lesão autoprovocada intencionalmente por disparo de outra arma de fogo e de arma de fogo não especificada" (X74), sendo 19,4\% em 2000, 10,0\% em 2009 e 4,6\% em 2018 e óbitos por "lesão autoprovocada intencionalmente por precipitação de um lugar elevado" (X80), 9,7\% em 2000, 12,5\% em 2009 e 13,8\% em 2018. Do total (2000-2018) $55,6 \%$ realizaram-se por "lesão autoprovocada intencionalmente por enforcamento, estrangulamento e sufocação", $20,6 \%$ item "outros", $11,9 \%$ por "lesão autoprovocada intencionalmente por disparo de outra arma de fogo e de arma de fogo não especificada" e $11,8 \%$ por "lesão autoprovocada intencionalmente por precipitação de um lugar elevado" (Figura 2).

Em relação aos locais de ocorrência dos óbitos, o domicílio apresentou-se como sendo o mais frequente, tendo ocorrido em $58,1 \%$ dos casos em 2000, 62,5\% em 2009 e 73,8\% em 2018. Na sequência vieram os hospitais, sendo $25,8 \%$ em 2000, 22,5\% em 2009 e $7,7 \%$ em 2018. No total (entre os anos de $2000-2018$ ), $58,0 \%$ dos óbitos ocorreram nos domicílios, $19,4 \%$ nos hospitais, $11,9 \%$ em vias públicas (Figura 3).

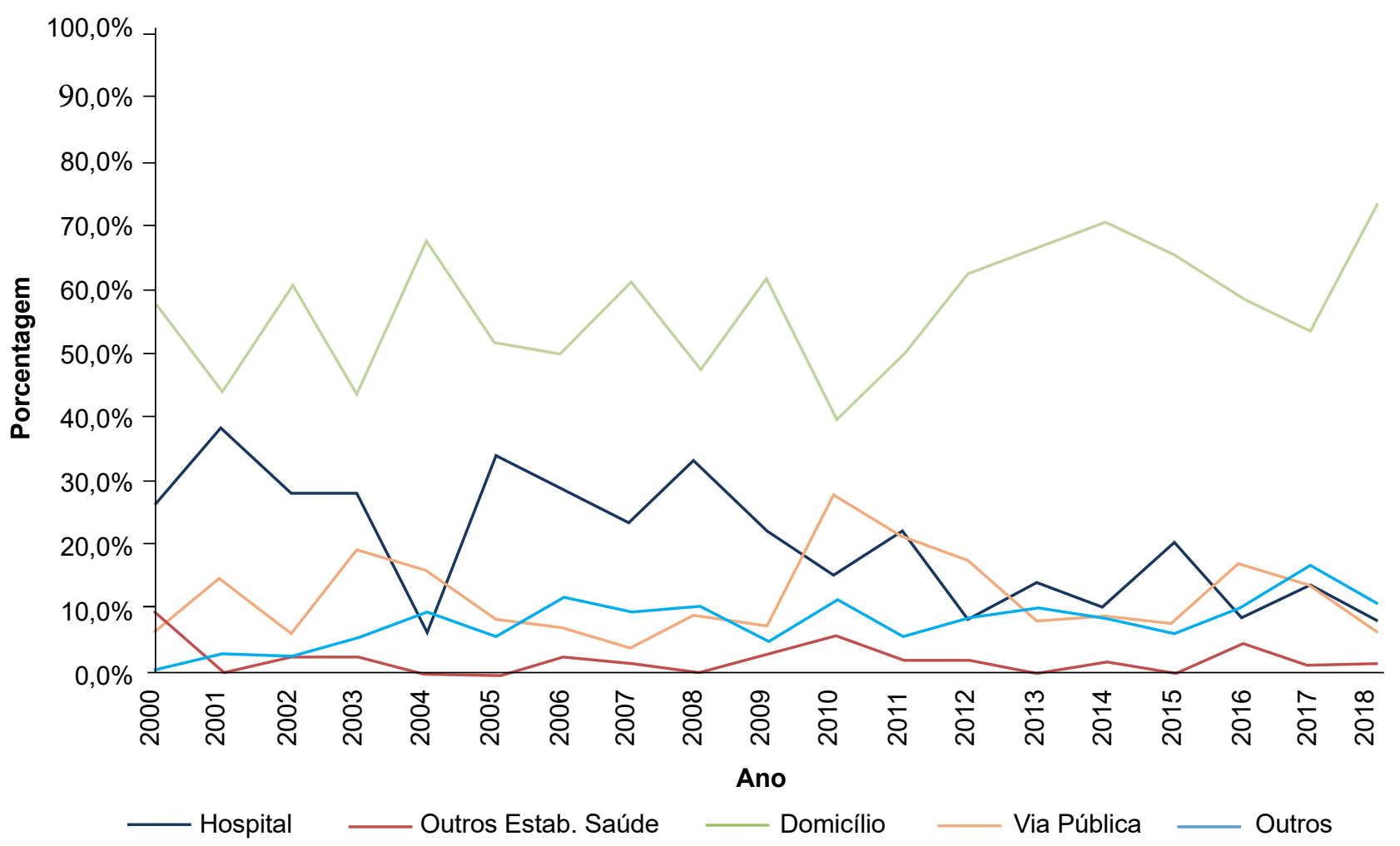

Fonte: SIM: Sistema de Informação sobre Mortalidade, TABNET- SMS. Campinas, São Paulo.

Figura 3 - Porcentagem de óbitos por suicídio na cidade de Campinas, São Paulo, em função do local de ocorrência e do ano (2000 a 2018). Campinas, São Paulo, 2019.

No total dos anos do estudo (2000-2018) os meses de maior ocorrência dos óbitos aconteceram entre setembro $(10,3 \%)$ e dezembro $(10,2 \%)$, e os de menor frequência aconteceram entre abril $(5,5 \%)$ e junho $(6,7 \%)$, porém esta observação não se apresenta uniforme para todos os anos. Tomando o início (2000), o meio (2009) e o final (2018) da série histórica, os meses de maior frequência apresentaram-se em 2000 , junho (22,6\%) e maio (16,1\%); para 2009, janeiro (17,5\%) e dezembro (15,0\%), para 2018, setembro (13,8\%) e outubro $(13,8 \%)$. Já os meses de menor frequência para 2000 , abril (sem casos), julho $(3,2 \%)$, agosto $(3,2 \%)$ e novembro $(3,2 \%)$, para 2009 , julho (sem casos) e março $(5,0 \%)$, maio $(5,0 \%)$ e agosto $(5,0 \%)$ e para 2018 , março $(4,6 \%)$, maio $(4,6 \%)$ e junho $(4,6 \%)$.

\section{DISCUSSÃO}

Dentre as causas externas de violência autoprovocadas, o suicídio está entre as três principais causas de mortalidade ${ }^{(1)}$. Na cidade de Campinas, em São Paulo, observou-se no presente estudo o aumento significativo 
nas taxas de suicídios no período de 2000 (3,20/100.000 hab.) a 2018 (5,42/100.000 hab.), cujas vítimas constataram-se como sendo predominantemente homens, adultos, de raça/cor branca, que escolheram como meio o enforcamento, realizado em domicílio.

O suicídio é um problema global de saúde pública. Dados mundiais mostram um aumento de $60 \%$ nos últimos 45 anos com tendência de crescimento nas próximas décadas ${ }^{(8)}$. Segundo estimativas da OMS a taxa global de suicídio padronizada por idade para 2016 apresentou-se como de 10,5 por cada 100.000 pessoas, variando amplamente entre os países, de cinco mortes por suicídio por cada 100.000 a mais de $30 / 100.000^{(3)}$.

Observou-se no atual estudo o aumento significativo do suicídio, no decorrer do tempo, para o sexo masculino com taxa de 5,30/100.000 hab. em 2000 e 8,45/100.000 hab. em 2018; dado este também descrito nas estimativas da OMS em 2019, que relatou agravo 1,8 vezes maior em homens quando comparado às mulheres ${ }^{(3)}$. No Brasil, vários estudos apontam maior percentual de óbitos entre a população masculina como, por exemplo: Bahia: $85,4 \%{ }^{(23)}$; Roraima:74,1\%(24); Sergipe: 74,4\%(25); Espirito Santo: 73,5\% ${ }^{(26)}$; Rio Grande do Sul:79,4\% ${ }^{(27)}$; Morada Nova, Ceará: $76,1 \%{ }^{(28)}$; São Luís, Maranhão: $81,8 \%{ }^{(29)}$. Já observando por regiões do Brasil, tem-se: Norte: $78,7 \%$, Nordeste:78,7\%, Centro-Oeste: $78,1 \%$, Sudeste: $78,7 \%$ e Sul:80,3\% ${ }^{(30)}$. O mesmo de observa em outros continentes como: Asiático

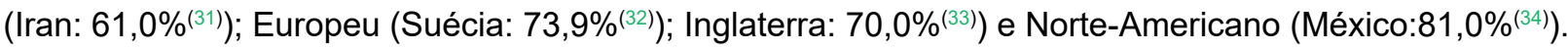

Situações de crise econômica, desemprego, bem como, a associação do homem como provedor familiar, podem levar a situações de vulnerabilidade familiar, isolamento, aumento do consumo de álcool e drogas e impulsividade, e demais fatores que podem contribuir para deixar o homem mais exposto ao risco de suicídio ${ }^{(1,35)}$. A menor ocorrência de suicídio entre as mulheres tem sido atribuída à maior busca de apoio espiritual ${ }^{(1)} \mathrm{e}$ o reconhecimento precoce dos sinais de risco para comportamento suicida, especialmente durante episódios agudos de transtornos psiquiátricos, buscando apoio nas redes de saúde ${ }^{(28)}$.

Embora os idosos sejam os mais acometidos pelo agravo(2,6), observou-se aumento significativo de óbitos por suicídio nos indivíduos de 20 a 60 anos incompletos. Estudos revelam ser o adulto jovem e os indivíduos de meia idade, os grupos mais expostos ao agravo. Assim temos os dados de: Roraima (mediana de idade 29 anos - não indígenas e 24 anos-indígenas) ${ }^{(24)}$; Sergipe (76,6\% de 20 a 59 anos) ${ }^{(25)}$; Rio Grande do Sul (10,3\% de 50 a 59 anos ${ }^{(27)}$; Morada Nova, Ceará (32,8\% de 40 a 49 anos) ${ }^{(28)}$; Iran (média de 32 anos) ${ }^{(31)}$; México $(37,2 \% \text { de } 30 \text { a } 49 \text { anos) })^{(34)}$.

Observou-se no presente estudo o maior percentual de óbitos entre a população branca (74,9\%), característica esta, também relatada em estudo realizado no Sul do país, como por exemplo, Santa Catarina: $90 \%$ (36); já o boletim epidemiológico publicado em $2017^{(6)}$, aponta a população indígena como a de maior taxa de suicídio no Brasil $\left(15,2 / 100.000\right.$ hab.), e $2019^{(5)}$ (pardos/negros - 54\%). Outro dado a ser observado é o fato da população do estado de São Paulo apresentar o número de $63,9 \%$ de pessoas brancas, segundo pesquisa feita pelo Censo 2010 . Ou seja, compreende-se que esta variável deve ser analisada com cuidado, uma vez que entre regiões há diferenças importantes entre raças.

A maioria dos óbitos por suicídio na atual investigação ocorreu na residência $(58,0 \%)$, sendo o enforcamento o meio mais utilizado $(55,6 \%)$, seguido por disparo de outra arma de fogo e precipitação de um lugar elevado. $O$ suicídio por enforcamento explicitou-se também como o principal meio em estudos nacionais e internacionais com as taxas de: Bahia $(64,3 \%)^{(23)}$; Roraima $(86,9 \%)^{(24)}$; Sergipe $(54,7 \%)^{(25)}$; Rio Grande do Sul $(72,5 \%)^{(27)}$; Morada Nova, Ceará $(49,2 \%)^{(28)}$; São Luís, Maranhão $(41,2 \%)^{(29)}$; Santa Catarina $(76 \%$ para os homens e $63 \%$ para as mulheres $63 \%)^{(36)}$; Inglaterra $(63,0 \%)^{(33)}$ e México $(78,8 \%)^{(34)}$. Compreende-se que por ser um meio de morte autoprovocada de fácil acesso, o controle por enforcamento é difícil. Por isso, faz-se importante identificar precocemente as pessoas com risco, e orientar familiares e amigos próximos para uma vigília mais constante da identificação de momentos emergenciais com intuito de impedir tal agravo ${ }^{(37)}$.

Também se observou a variação significativa nas porcentagens de morte em função do mês do ano na presente pesquisa, com aumento dos casos (2000 - 2018) em setembro e dezembro. No entanto, esses dados divergem dos apresentados em estudo realizado em duas regiões do estado de São Paulo, com menor predomínio de óbitos por suicídio entre os meses pertencentes â primavera (15,6\%) (setembro e dezembro) $)^{(38)}$.

Para cada morte por suicídio estima-se a ocorrência de mais de 20 tentativas, sendo o histórico de tentativa anterior um preditor de morte pelo agravo ${ }^{(8,12)}$, portanto, compreende-se que é um evento que pode ser evitável, desde que seja detectado precocemente e receba intervenção imediata adequada (ressaltando-se que os esforços para a promoção da saúde e a prevenção do comportamento suicida extrapolam os conhecimentos do campo da saúde ${ }^{(2)}$ ). Dessa forma, por ser um fenômeno complexo e multifatorial, essas ações devem ser fundamentadas nos fatores de risco e proteção, bem como, no conhecimento produzido por profissionais de diversas áreas do saber ${ }^{(2)}$.

Atualmente, a problemática do suicídio está mais presente e novos desafios serão impostos a todos os envolvidos com a promoção da saúde mental da população, levando a discussões sobre a natureza e os determinantes da 
promoção da saúde mental a nível populacional e institucional. A pandemia COVID-19 provocada pelo novo coronavírus SARS-COV-2, ocasionou perturbações psicológicas e sociais que afetam a capacidade de enfrentamento de toda a sociedade. Essas perturbações podem favorecer o surgimento ou agravamento dos sintomas psiquiátricos principalmente em pessoas com vulnerabilidade específica a transtornos mentais, aumento do uso de substâncias e dependência de álcool( ${ }^{(39)}$, por exemplo, que são fatores de risco para o suicídio ${ }^{(2,11)}$. Embora o impacto da pandemia COVID-19 para as doenças psíquicas ainda esteja sendo mensurado, há indícios preocupantes do aumento do sofrimento psicológico, dos sintomas psíquicos, transtornos mentais e do trauma psicológico provocados diretamente pela infecção ou por seus desdobramentos secundários. Portanto, esforços imediatos devem ser empregados, em todos os níveis e pelas diversas áreas de conhecimento, a fim de minimizar resultados ainda mais negativos na saúde mental da população ${ }^{(39)}$. As ações de promoção da saúde terão um papel central a desempenhar no empoderamento das comunidades e dos indivíduos na adoção de respostas eficazes e na gestão dos impactos psicossociais das consequências multifacetadas da pandemia ${ }^{(39)}$.

Não obstante às contribuições feitas nesta pesquisa, algumas limitações devem ser consideradas, como os dados obtidos de fontes secundárias que podem estar sujeitos à falta de padronização na coleta de dados, além de lacunas de informações que podem ser importantes para as análises de interesse e a variação de cobertura conforme tempo e espaço ${ }^{(4)}$. Em se tratando de suicídio, a subnotificação é outra limitação; pois em função do estigma social relacionado ao agravo, sendo, muitas vezes, ocultado e registrado como, por exemplo, mortes de intenção indeterminada, acidentes, homicídios, causas desconhecidas, afogamentos, e envenenamentos acidentais; o que acarreta prejuízo no reconhecimento do panorama real da magnitude do fenômeno ${ }^{(2,28)}$. A despeito das limitações supracitadas, deve-se ressaltar que estudos de séries temporais podem ser importantes ferramentas epidemiológicas para a formulação de hipóteses explicativas e avaliação indireta da efetividade das políticas públicas.

\section{CONCLUSÃO}

Os resultados do presente estudo documentaram não só o crescente aumento das taxas de suicídios no município de Campinas, em São Paulo, no período analisado (2000 - 2018), como também o perfil de suas vítimas, cuja predominância mostrou-se como sendo do sexo masculino, adultos, de raça/cor branca, que escolheram como meio o enforcamento, realizado em domicílio, nos meses de setembro e dezembro.

\section{CONFLITOS DE INTERESSE}

Os autores declaram não existirem conflitos de interesses.

\section{CONTRIBUIÇÕES}

Natalia Cristina da Silva Freire contribuiu com a elaboração e delineamento do estudo; aquisição, análise e interpretação de dados. Taís Daiene Russo Hortencio contribuiu com a redação e a revisão do manuscrito. Márcia Regina Campos Costa da Fonseca contribuiu com a elaboração e delineamento do estudo; a aquisição, análise e interpretação de dados; e a redação e revisão do manuscrito. Todas as autoras aprovaram a versão final a ser publicada.

\section{REFERÊNCIAS}

1. World Health Organization. Preventing suicide: a global imperative [Internet]. Geneva: World Health Organization; 2014 [acesso em 2020 Abr 17]. Disponível em: www.who.int/mental_health/suicide-prevention/ world_report_2014/en/

2. Teixeira SMO, Souza LEC, Viana LMM. O suicídio como questão de saúde pública. Rev Bras Promoç Saúde. 2018 [acesso em 2020 Abr 17];31(3):1-3. doi: 10.5020/18061230.2018.8565

3. World Health Organization. Suicide in the world: global health estimates [Internet]. Geneva: World Health Organization; 2019 [acesso em 2020 Jun 26]. Disponível em: www.who.int/mental_health/prevention/suicide/ suicideprevent/en/

4. Garbin CAS, Santos LFP, Moimaz SAS, Saliba O. A operacionalização do SUS na prevenção e condução de casos de suicídios: análise documental. Rev Ciênc Plur [Internet]. 2019 [acesso em 2020 Jun 26];5(2):12942. doi: $10.21680 / 2446-7286.2019 v 5 n 2 I D 17528$ 
5. Ministério da Saúde (BR). Perfil epidemiológico dos casos notificados de violência autoprovocada e óbitos por suicídio entre jovens de 15 a 29 anos no Brasil, 2011 a 2018. Bol Epidemiol [Internet]. 2019 [acesso em 2021 Abr 12];50(24):1-14. Disponível em: https://portalarquivos2.saude.gov.br/images/pdf/2019/setembro/13/ BE-suic--dio-24-final.pdf

6. Ministério da Saúde (BR). Suicídio: saber, agir e prevenir. Bol Epidemiol [Internet]. 2017 [acesso em 2020 Abr 17];48(30):1-14. Disponível em: https://www.saude.gov.br/images/pdf/2017/setembro/21/2017-025-Perfilepidemiologico-das-tentativas-e-obitos-por-suicidio-no-Brasil-e-a-rede-de-atencao-a-saude.pdf

7. Ministério da Saúde (BR), Departamento de Informática do Sistema Único de Saúde. Sistema de informação sobre mortalidade [Internet]. [data desconhecida] [acesso em 2021 Abr 12]. Disponível em: http://tabnet. datasus.gov.br/cgi/deftohtm.exe?sim/cnv/ext10br.def

8. World Health Organization. Comprehensive Mental Health Action Plan 2013-2020 [Internet]. Geneva: World Health Organization; 2013 [acesso em 2020 Abr 17]. Disponível em: http://apps.who.int/gb/ebwha/pdf_files/ WHA66/A66_R8-en.pdf

9. Rodrigues CD, Souza DS, Rodrigues HM, Konstantyner TCRO. Trends in suicide rates in Brazil from 1997 to 2015. Braz J Psychiatry [Internet]. 2019 [acesso em 2020 Jun 26];41(5):380-8. doi: 10.1590/1516-4446-20180230

10. Dantas ESO. Prevenção do suicídio no Brasil: como estamos? Physis Rev Saúde Colet [Internet]. 2019 [acesso em 2020 Jun 26];29(3):e290303. doi: 10.1590/s0103-73312019290303

11. Organização Mundial de Saúde. Prevenção do suicídio: um manual para profissionais da saúde em atenção primária [Internet]. Genebra: Organização Mundial da Saúde; 2000 [acesso em 2020 Abr 17]. Disponível em: http://bvsms.saude.gov.br/bvs/publicacoes/atencaobasica.pdf

12. Bachmann S. Epidemiology of Suicide and the Psychiatric Perspective. Int J Environ Res Public Health [Internet]. 2018 [acesso em 2020 Jun 26];15(7):1425. doi: 10.3390/ijerph15071425

13. Ministério da Saúde (BR). Portaria $n^{\circ} 1.876$, de 14 de agosto de 2006. Institui Diretrizes Nacionais para Prevenção do Suicídio, a ser implantadas em todas as unidades federadas, respeitadas as competências das três esferas de gestão [Internet]. Brasília: Ministério da Saúde; 2006 [acesso em 2020 Jun 26]. Disponível em: http://bvsms.saude.gov.br/bvs/saudelegis/gm/2006/prt1876_14_08_2006.html

14. Secretaria Municipal de Saúde de Campinas. Sistema de Informações em Saúde [Internet]. [data desconhecida] [acesso em 2019 Jul 9]. Disponível em: http://www.saude.campinas.sp.gov.br/saude/tabnethome/index.htm

15. Instituto Brasileiro de Geografia e Estatística. Campinas-Panorama [Internet]. 2021 [acesso em 2021 Abr 10]. Disponível em: https://cidades.ibge.gov.br/brasil/sp/campinas/panorama

16. Organização Mundial da Saúde. CID-10 Classificação Estatística Internacional de Doenças e Problemas Relacionados à Saúde. São Paulo: Universidade de São Paulo; 1997.

17. Gonçalves JP. Ciclo vital início, desenvolvimento e fim da vida humana possíveis - contribuições para educadores. Contexto Educ [Internet]. 2016 [acesso em 2020 Jun 26];31(98):79-110. doi: 10.21527/21791309.2016.98.79-110

18. Rede Interagencial de Informação para a Saúde. Indicadores básicos para a saúde no Brasil: conceitos e aplicações [Internet]. Brasília: Organização Pan-Americana da Saúde; 2008 [acesso em 2021 Abr 12]. Disponível em: http://www.ripsa.org.br/2014/10/30/indicadores-basicos-para-a-saude-no-brasil-conceitos-eaplicacoes-livro-2a-edicao-2008-2/

19. Rouquayrol MZ, Almeida N Filho. Epidemiologia \& Saúde. 6ª ed. Rio de Janeiro: MEDSI; 2003.

20. Ministério da Saúde (BR), Departamento de Informática do Sistema Único de Saúde. Informações de Saúde: demográficas e Socioeconômicas [Internet]. [data desconhecida] [acesso em Jul 09]. Disponível em: http:// tabnet.datasus.gov.br/cgi/deftohtm.exe?ibge/cnv/popuf.def

21. Morettin PA, Bussab WO. Estatística básica. São Paulo: Saraiva Educação SA; 2017.

22. Core Team. R: A language and environment for statistical computing. R Foundation for Statistical Computing, Vienna, Austria [Internet]. 2018 [acesso em 2020 Mar 2]. Disponível em: https://www.R-project.org/ 
23. Carmo EA, Santos PHS, Ribeiro BS, Soares CJ, Santana MLAA, Bomfim ES, et al. Características sociodemográficas e série temporal da mortalidade por suicídio em idosos no estado da Bahia, 19962013. Epidemiol Serv Saude [Internet]. 2018 [acesso em 2020 Jun 26];27(1):1-8. doi: 10.5123/S167949742018000100001

24. Souza MLP, Onety RTS Jr. Caracterização da mortalidade por suicídio entre indígenas e não indígenas em Roraima, Brasil, 2009-2013. Epidemiol Serv Saude [Internet]. 2017 [acesso em 2020 Jun 26];26(4):887-93. doi: 10.5123/s1679-49742017000400019

25. Santos AD, Guimarães LML, Carvalho YF, Viana LC, Alves GL, Lima ACR, et al. Análise espacial e tendência temporal da mortalidade por suicídio em Sergipe, Brasil, 2000-2015. Trends Psychiatry Psychother [Internet]. 2018 [acesso em 2020 Jun 26];40(4):269-76. doi: 10.1590/2237-6089-2017-0028

26. Tavares FL, Borgo VMP, Leite FMC, Cupertino EGF, Pereira JÁ, Alves RNR, et al. Mortalidade por suicídio no Espírito Santo, Brasil: análise do período de 2012 a 2016. Av Enferm [Internet]. 2020 [acesso em 2020 Jun 26];38(1):66-76. doi: 10.15446/av.enferm.v38n1.79960

27. Franck MC, Monteiro MG, Limberger RP. Mortalidade por suicídio no Rio Grande do Sul: uma análise transversal dos casos de 2017 e 2018. Epidemiol Serv Saude [Internet]. 2020 [acesso em 2020 Jun 26];29(2):e2019512. doi: 10.5123/s1679-49742020000200014

28. Souza SA Jr, Rodrigues CF. Mortalidade por suicídio: realidade de uma cidade no interior do nordeste brasileiro. Rev Bras Promoç Saúde [Internet]. 2020 [acesso em 2020 Jun 26];33:10813. doi: 10.5020/18061230.2020.10813

29. Ramos ASMB, Nunes MJ, Almeida HFR, Gouveia DM, Furtado DRL, Mourão MHV. Ocorrência de suicídio na ilha de São Luís entre os anos de 2012-2016. Revista Nursing [Internet]. 2019 [acesso em Abr 12];22(251):2932-6. Disponível em: http://www.revistanursing.com.br/revistas/251/pg117.pdf

30. D'Eça A Jr, Rodrigues LS, Meneses EP Filho, Costa LDLN, Rêgo AS, Costa LC, et al. Mortalidade por suicídio na população brasileira, 1996-2015: qual é a tendência predominante? Cad Saúde Colet [Internet]. 2019 [acesso em 2020 Jun 26];27(1):20-4. doi: 10.1590/1414-462X201900010211

31. Kangavari HN, Shojaei A, Nazari SSH. Suicide mortality trends in four provinces of Iran with the highest mortality, from 2006-2016. J Res Health Sci [Internet]. 2017 [acesso em 2020 Jun 5];17(2):1-9. Disponível em: http://jrhs.umsha.ac.ir/index.php/jrhs/article/view/3149/pdf

32. Lageborn CT, Ljung R, Vaez M, Dahlin M. Ongoing university studies and the risk of suicide: a register-based nationwide cohort study of 5 million young and middle-aged individuals in Sweden, 1993-2011. BMJ Open [Internet]. 2017 [acesso em 2020 Jun 26];7(3). doi: 10.1136/bmjopen-2016-014264

33. Rodway CMA, Tham SG, Ibrahim S, Turnbull P, Windfuhr K, Shaw J, et al. Suicide in children and young people in England: a consecutive case series. Lancet Psychiatry [Internet]. 2016 [acesso em 2020 Jun 26];3(8):751-9. doi: 10.1016/S2215-0366(16)30094-3

34. Dávila-Cervantes CA. Factores sociodemográficos asociados a la mortalidad por suicidios en México, 20122016. Univ Salud [Internet]. 2019 [acesso em 2020 Jun 26];21(3):235-9. doi: 10.22267/rus.192103.160

35. Marín-León L, Barros MBA. Mortes por suicídio: diferenças de gênero e nível socioeconômico. Rev Saúde Pública [Internet]. 2003 [acesso em 2020 Jun 26];37(3):357-63. doi: 10.1590/S0034-89102003000300015

36. Benetti IC, Molina LR, Kornin A. Características do suicídio em Santa Catarina: um estudo do período de 2007 a 2016. Estud Psicol [Internet]. 2018 [acesso em 2020 Jun 26];23(40):404-15. doi: 10.22491/16784669.20180038

37. Machado DB, Santos DN. Suicídio no Brasil, de 2000 a 2012. J Bras Psiquiatr [Internet]. 2015 [acesso em 2020 Jun 26];64(1):45-54. doi: 10.1590/0047-2085000000056

38. Baptista MN, Carneiro AM, Gomes JO, Cardoso HF. Análise Epidemiológica do Suicídio em duas Regiões do Estado de São Paulo entre 2004 e 2008. Psicol Pesq [Internet]. 2012 [acesso em 2020 Jun 10];6(1):2-12. Disponível em: http://pepsic.bvsalud.org/pdf/psipesq/v6n1/v6n1a02.pdf

39. Vigo D, Patten S, Pajer K, Krausz M, Taylor S, Rush B, et al. Mental health of communities during the COVID-19 pandemic. Can J Psychiatry [Internet]. 2020 [acesso em 2020 Jun 26];11;65(10):681-7. doi: $10.1177 / 0706743720926676$ 
40. Coeli CM. Sistemas de Informação em Saúde e uso de dados secundários na pesquisa e avaliação em saúde. Cad Saúde Colet [Internet]. 2010 [acesso em 2021 Abr 12];18(3):335-6. Disponível em: http://www. cadernos.iesc.ufrj.br/cadernos/images/csc/2010_3/artigos/CSCv18n3_pag335-6.pdf

\section{Endereço do primeiro autor:}

Natalia Cristina da Silva Freire

Faculdade São Leopoldo Mandic

Rua Dr. José Rocha Junqueira, 13

Bairro Ponte Preta

CEP: 13045-755 - Campinas - SP - Brasil

E-mail: ncfreire94@gmail.com

\section{Endereço para correspondência:}

Márcia Regina Campos Costa da Fonseca.

Faculdade São Leopoldo Mandic

Rua Dr. José Rocha Junqueira, 13

Bairro Ponte Preta

CEP:13045-755 - Campinas - SP - Brasil

E-mails: marcia.fonseca@sImandic.edu.br

Como citar: Freire NCS, Hortencio TDR, Fonseca MRCC. Série histórica de mortalidade por suicídio em município paulista segundo dados epidemiológicos. Rev Bras Promoç Saúde. 2021;34:11393. 